\title{
Effect of high-frequency radiations on survival of the honeybee (Apis mellifera L.)
}

\author{
Keyvin Darney ${ }^{1}$, Aurélie GIRAUdIN ${ }^{1}$, Romain JOSEPH $^{1}$, Pauline ABADIE ${ }^{1}$, \\ Pierrick Aupinel ${ }^{2}$, Axel Decourtye ${ }^{3,4}$, Eric Le Bourg ${ }^{1}$, Monique Gauthier ${ }^{1}$ \\ ${ }^{1}$ UMR CNRS 5169, Centre de Recherches sur la Cognition Animale, Université Paul Sabatier, 118 Route de Narbonne, \\ 31062, Toulouse cedex, France \\ ${ }^{2}$ INRA, Unité expérimentale d'entomologie Le Magneraud, 17700, Surgères, France \\ ${ }^{3}$ ACTA, UMT PrADE, 228 route de l'aérodrome, 84914, Avignon cedex 9, France \\ ${ }^{4}$ ITSAP-Institut de l'abeille, UMT PrADE, 228 route de l'aérodrome, 84914, Avignon cedex 9, France
}

Received 17 March 2015 - Revised 24 September 2015 - Accepted 27 November 2015

\begin{abstract}
Recent studies succeeded in developing a method to automatically record honeybees going in and out of the hive. Honeybees were individualized with radio frequency identification (RFID) tags glued onto their dorsal surface and detected at the hive entrance by readers emitting high-frequency (HF) radio waves. In this work we search for a possible adverse effect of HF on honeybees' survival. Eight-day-old honeybees were exposed to HF (13.56 MHz) or ultra-high-frequency (UHF, $868 \mathrm{MHz}$ ) radio waves for $2 \mathrm{~h}$ split into ON and OFF periods. The ON/ OFF ratio was 1:3 (OFF duration 3, 90, 180, 370 and $360 \mathrm{~s}$ ) or 1:5 (OFF duration $300 \mathrm{~s}$ ). Dead individuals were counted every day, and the cumulative mortality rates of exposed and non-exposed honeybees were compared 7 days after exposure. Out of the five experimental conditions, we observed an increase in mortality in two conditions, once after HF and once after UHF exposure, with OFF duration of $5 \mathrm{~min}$ or more. We then recommend limiting exposure of honeybees to radio waves to less than $2 \mathrm{~h}$ per day, and we conclude that the RFID parameters, like those we used in the field for monitoring hive activity, present no adverse effects for honeybees.
\end{abstract}

\section{electromagnetic radiation / environment / health / insect}

\section{INTRODUCTION}

Evaluating the activity of the hive is a major challenge for beekeepers and scientists. This activity reflects the behaviour and health of the colony and can reveal poisoning situations induced by insecticide treatments applied to plants visited by honeybees or by in-hive treatment to fight against diseases or parasites. Automatic recording and identification of individual eusocial insects are possible using radio frequency identification (RFID) device (Streit et al. 2003; Sumner et al. 2007; Molet et al. 2008; Stelzer and Chittka

Corresponding author: M. Gauthier, monique.gauthier@univ-tlse3.fr

Manuscript Editor: Yves Le Conte
2010). Each insect tagged with a tiny electronic transponder is detected each time it enters or leaves the nest thanks to RFID readers located at the nest entrance. Recent ecotoxicological studies succeeded in recording several bees to define the number of their foraging flights. Such an experimental setup has been successfully used to study the adverse effect of pesticides on foraging activity and homing flight (Decourtye et al. 2011; Feltham et al. 2014; Gill et al. 2012; Gill and Raine 2014; Henry et al. 2012; Henry et al. 2014; Schneider et al. 2012).

The currently used RFID antennae emit highfrequency radio waves $(\mathrm{HF} ; 13.56 \mathrm{MHz}$ ) that require a very short reading distance of $2 \mathrm{~mm}$ between the readers and the tagged honeybee to detect the electronic tag glued onto the dorsal surface of the honeybee. A new reader generation 
emitting ultra-high-frequency (UHF; $868 \mathrm{MHz}$ ) radio waves, similar to the one used by the Global System for Mobile telecommunication (GSM, $900 \mathrm{MHz}$ ), can be used with a longer reading distance. The 13.56- and $868 \mathrm{-MHz}$ frequencies are lower than the $2.4-\mathrm{GHz}$ frequency used in microwave ovens and are not supposed to induce heating in insects. Radio waves are received by the insect each time it goes beneath the readers. Data collected from our previous RFID field experiments (Decourtye et al. 2011) indicate that each tagged honeybee can be detected at the most 100 times per day over a 15-day period. Each passage beneath the readers was estimated at $2 \mathrm{~s}$ leading to a cumulative exposure of almost $1 \mathrm{~h}$ (exactly $50 \mathrm{~min}$ ) for a duration of observation of 15 days. We then wonder whether radio wave exposure was safe for honeybees, because this is a condition for using the RFID technique for routine monitoring of the activity of the hive.

Exposure to radio waves has hugely increased over the last 10 years owing to the increasing use of wireless communication. Numerous studies are devoted to depicting the relationship between radio waves and alterations of biological and behavioural functions or their ecological effects (for review, see Cucurachi et al. 2013). Although a direct relationship has not been established between radio wave exposure and health problems (Krewski et al. 2007; Vanderstraetan and Verschaeve 2008), behavioural and spatial memory disorders in humans (D'Andrea et al. 2003; Wiholm et al. 2009) and orientation disorders in honeybees and birds (Warnke 2007) have been suspected and a negative effect of radio waves on the in-hive behaviour and homing flight of honeybees has been reported (Harst et al. 2006; Kimmel et al. 2007). Cell phone radiations were suspected of affecting the reproductive capacity of Drosophila (Panagopoulos et al. 2004) and the egg laying rate of the honeybees' queen (Sharma and Kumar 2010) and inducing the worker piping signal in the hive (Favre 2011).

The aim of our work is to make the use of RFID technique safe for recording hive activity by studying the effect of high and ultra-highfrequency (13.56 and $868 \mathrm{MHz}$, respectively) radio waves on honeybees' survival. Incidentally, we also contribute thereby to the study of the effects of radio waves on living organisms.

\section{MATERIAL AND METHODS}

Honeybees The experiments were performed in spring and summer time in Toulouse (south-western France) in 2 consecutive years and were conducted each year on a 10-comb Dadant $\odot$ hive comprising about 20,000 workers. Emergent honeybees were used. A brood frame picked up from the hive was maintained in an incubator, in controlled conditions $\left(33^{\circ} \mathrm{C}, 40 \%\right.$ relative humidity). Each day, honeybees were collected when emerging from the cells and were equally distributed in three polypropylene cages, one being assigned to the control (non-exposed) group, and the other ones to the HF and UHF groups. The number of honeybees gathered per cage depended on the number of honeybees collected each day and was not less than 20 .

Each cage $(12 \mathrm{~cm} \times 7 \mathrm{~cm} \times 8 \mathrm{~cm})$ was equipped with two feeder tubes $(1.5 \mathrm{ml})$ filled with a sugar solution $(40 \%$ $w / v)$. Feeder plates containing pollen and sugar paste (Apifonda, Ickowicz, Bollène, France) were placed inside the cage. The food was provided ad libitum and was renewed each day; pollen was available during the first week. To ensure aeration, small holes were pierced in the two largest sides of the cage. A small piece of honeycomb was put into the cage to mimic the hive environment. To collect excreta of the honeybees, the bottom of the cage was covered with an absorbent paper, renewed each time it was necessary. The cages were maintained in darkness in the incubator throughout the experiment. As adult bees are sufficiently mature to begin flying (Capaldi and Dyer 1999) and thus to be recorded by RFID readers 5 to 7 days after emergence, honeybees aged 8 days were taken off the incubator to be exposed to HF or UHF, in accordance with one of the conditions described below. At the end of the exposure period, the cages were replaced into the incubator for 7 days. Each day, dead honeybees were removed from the cages.

Radio wave exposure Two radio frequency generators $(22 \mathrm{~cm} \times 15 \mathrm{~cm} \times 6 \mathrm{~cm}$, Tag Tracing Solution, Valence, France) emitting high-frequency (13.56 MHz) or ultra-high-frequency $(868 \mathrm{MHz})$ radio waves were used. The power densities of HF and UHF radiations were measured with an electromagnetic field meter (Spectran HF-60105, AARONIA AG) at the distances 
of 0 and $8 \mathrm{~cm}$ above the generators (Table I). These distances correspond to the minimum $(0 \mathrm{~cm})$ and the maximum $(8 \mathrm{~cm})$ distances between the radiation source and the honeybees when the honeybee cages were placed on the generators. The power density value decreased to 0 at a distance of $10 \mathrm{~cm}$ above the HF generator and at a distance of about $20 \mathrm{~cm}$ above the UHF generator. We did not detect any radiation at a distance superior to $2 \mathrm{~cm}$ along the wall sides of both generators. These values indicate that the radio waves are emitted upwards by the generators on rather short distances.

The generators were placed into a Faraday cage (length $1.20 \mathrm{~m} \times$ width $1.00 \mathrm{~m} \times$ height $1.00 \mathrm{~m}$ ) put on the laboratory bench to avoid interference with electric devices present in the experimental room. The propagation of the radiations on short distances described above is an indication that the radio waves are probably not reflected by the Faraday cage. A distance of $50 \mathrm{~cm}$ was respected between the generators to avoid $\mathrm{HF}$ and UHF interferences. A third cage, serving as a control, was not exposed and was put inside the Faraday cage, at $50 \mathrm{~cm}$ from the two generators (Figure 1).

Each generator was connected to a computer controlling the onset, the offset and the cycle number of radio wave emissions (Tag Tracing Solution, Valence, France).

We have estimated that honeybees were exposed to high frequencies during RFID field experiments for $50 \mathrm{~min}$ (Decourtye et al. 2011). In the present experiments in the lab, we increased this value to $2 \mathrm{~h}$. During the field experiments, honeybees were exposed to HF for durations of less than $2 \mathrm{~s}$ each time they passed under the readers. To best mimic the field situation and taking into account the limits of the laboratory conditions, the 2-h exposure was divided into cycles of $\mathrm{ON}$ and $\mathrm{OFF}$ periods. The cycle number was defined so that the total exposure duration was $2 \mathrm{~h}$. Several ON durations were tested, with $\mathrm{OFF}$ duration greater than $\mathrm{ON}$ duration. The ON/OFF ratio was $1: 3$ and 1:5. The parameters of the protocols are gathered in Table II.

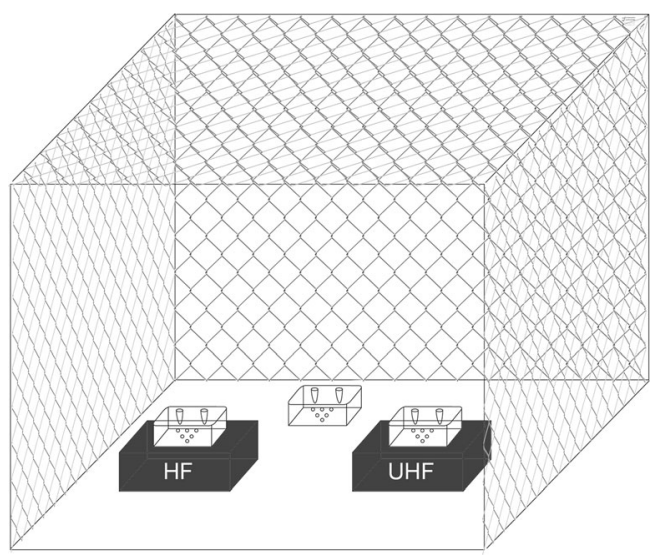

Figure 1. Experimental setup. The HF and UHF generators are put inside the Faraday cage and a cage of honeybees is placed on each generator. A third cage of honeybees for control is placed inside the Faraday cage.

Statistical analysis Cumulative mortality 7 days after exposure was analysed with a logistic model (DataDesk6.3 statistical package) taking into account the treatment (control, HF, UHF) and, for the experiments using the ON/OFF 1:3 ratio, the four conditions of exposure (Table II) and the interaction between these factors. Experiments using the 1:5 ratio were performed in 2 successive years, and mortality 7 days after exposure was analysed with a logistic model taking into account the treatment, the year of experiment and the interaction between these factors. Percentages of mortality for each condition are graphically represented with confidence intervals at the $P=0.05$ level in Figure 2.

\section{RESULTS}

During the exposure to the radio waves and each day of the week following the exposure, we

Table I. Power densities and field strength of HF and UHF radiations measured at 0 and $8 \mathrm{~cm}$ from the source.

\begin{tabular}{|c|c|c|c|c|}
\hline \multirow[t]{2}{*}{ Distance $(\mathrm{cm})$} & \multicolumn{2}{|l|}{ Power density } & \multicolumn{2}{|c|}{ Field strength } \\
\hline & $13.56 \mathrm{MHz}$ & $868 \mathrm{MHz}$ & $13.56 \mathrm{MHz}$ & $868 \mathrm{MHz}$ \\
\hline 0 & $42.54 \mathrm{nW} / \mathrm{m}^{2}$ & $65.35 \mathrm{~mW} / \mathrm{m}^{2}$ & $4.04 \mathrm{mV} / \mathrm{m}$ & $5 \mathrm{~V} / \mathrm{m}$ \\
\hline 8 & $3 \mathrm{nW} / \mathrm{m}^{2}$ & $31.01 \mathrm{~mW} / \mathrm{m}^{2}$ & $1.06 \mathrm{mV} / \mathrm{m}$ & $3.42 \mathrm{~V} / \mathrm{m}$ \\
\hline
\end{tabular}


Table II. Parameters of radio wave exposure for the five experimental conditions.

\begin{tabular}{llllllll}
\hline Conditions & ON/OFF & ON (s) & OFF (s) & $\begin{array}{l}\text { Cycle } \\
\text { number }\end{array}$ & $\begin{array}{l}\text { Time in the } \\
\text { Faraday cage (h) }\end{array}$ & $n(\mathrm{c}, \mathrm{HF}, \mathrm{UHF})$ & Replicates \\
\hline 1 & $1 / 3$ & 1 & 3 & 7200 & 8 & $158,228,190$ & 4 \\
2 & $1 / 3$ & 30 & 90 & 240 & 8 & $266,345,340$ & 3 \\
3 & $1 / 3$ & 60 & 180 & 120 & 8 & $125,176,177$ & 2 \\
4 & $1 / 3$ & 120 & 360 & 60 & 8 & $193,200,226$ & 3 \\
5 & $1 / 5$ & 60 & 300 & 120 & 12 & $193,200,200$ & 8 \\
\hline
\end{tabular}

For each condition, the numbers of animals $(n)$ are indicated for control (c), HF and UHF groups. The experiments of the conditions 1-4 were performed during successive weeks of June and July 2010. The experiments corresponding to the condition 5 were performed on 2 consecutive years (2009 and 2010) during late July

observed the honeybees in the cages and did not detect any abnormal behaviour. The honeybees exposed to radio waves did not move to the upper part of the box to get away from the radiation source; they occupied the whole volume of the box, as did the control honeybees. They did not present increased or decreased activity or aggressiveness, they did not emit any peculiar sounds and their consumption of water or food was equivalent to those of control bees.

Being subjected to HF and UHF radio waves with an ON/OFF ratio of 1:3 had no significant effect on mortality (Figure 2, F(2, 2612) $=0.64$; not significant (n.s.)). However, the percentages

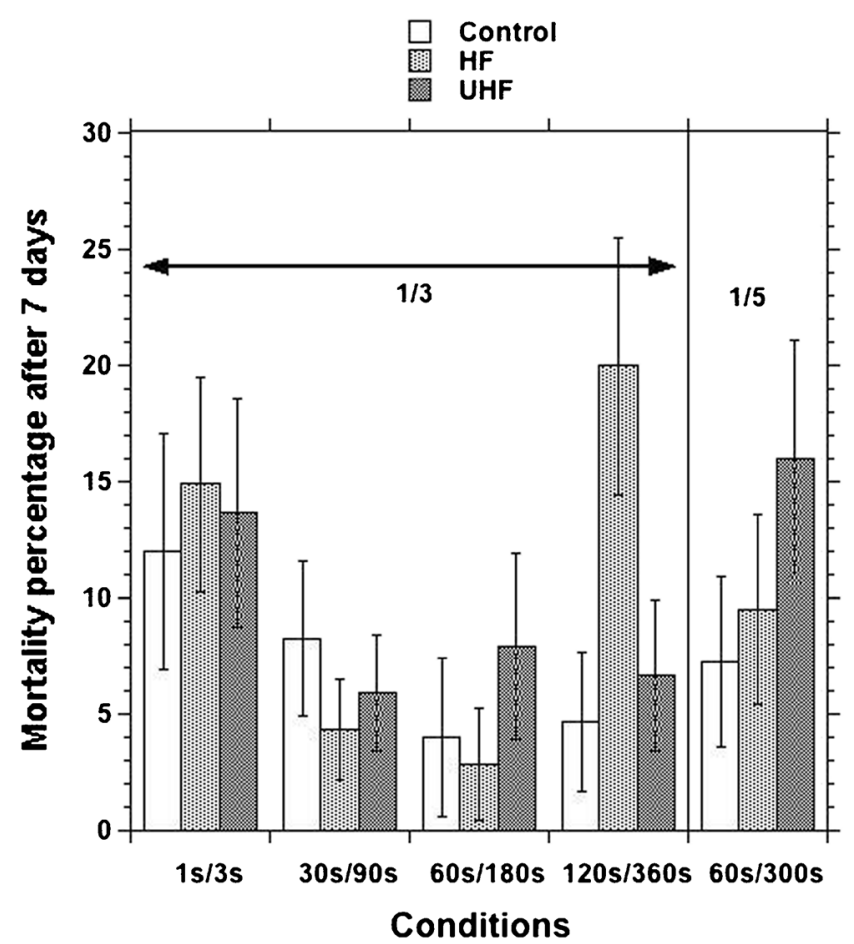

Figure 2. Mortality percentages ( \pm confidence interval at the $P=0.05$ level) 7 days after exposure to HF and UHF and in the control groups in the 1:3 and 1:5 conditions. For each bar, the number of bees for, respectively, control, HF and UHF groups is for $1 \mathrm{~s} / 3 \mathrm{~s}$ condition 158, 228 and 190; for $30 \mathrm{~s} / 90 \mathrm{~s}$ condition 266, 345 and 340; for $60 \mathrm{~s} / 180 \mathrm{~s}$ condition 125, 176 and 177; for $120 \mathrm{~s} / 360 \mathrm{~s}$ condition 193, 200 and 226; and for $60 \mathrm{~s} / 300 \mathrm{~s}$ condition 193, 200, 200. 
of mortality were linked to the condition of exposure $(\mathrm{F}(3,2612)=11.08 ; P \leq 0.0001)$, with the groups of the $1 \mathrm{~s} / 3 \mathrm{~s}$ condition displaying a higher mortality than both $30 \mathrm{~s} / 90 \mathrm{~s}$ and $60 \mathrm{~s} / 180 \mathrm{~s}$ groups (post hoc tests). This could be linked to seasonal or biological variations in the natural mortality of bees because the four conditions of the $1: 3$ ratio were performed in 4 successive weeks in June and July, with higher mortality early June. Finally, the significant interaction between condition and treatment $(\mathrm{F}(6,2612)=5.39$; $P \leq 0.0001)$ showed that the HF group of the $120 \mathrm{~s} / 360 \mathrm{~s}$ condition displayed a higher mortality than both control and UHF groups of this $120 \mathrm{~s} /$ 360 s condition (post hoc test), while no such effect was observed in the other conditions.

The treatment had a significant effect on mortality in the $60 \mathrm{~s} / 300 \mathrm{~s}$ condition (Figure 2, F(2, $587)=4.23, P=0.0151$ ), with the UHF group displaying a higher mortality than both $\mathrm{HF}$ and control groups (post hoc test). A higher mortality was observed during the second year of experiment for the HF, UHF and control groups $(\mathrm{F}(1$, $587)=17.41, P<0.0001 ; 7.05$ vs $18.90 \%$ ), but the interaction with the treatment was not significant $(F(2,587)=0.73$, n.s. $)$, showing that the treatment had similar effects in both years.

\section{DISCUSSION}

In our experiments, the duration of exposure to HF or UHF was constant and fixed at $2 \mathrm{~h}$, and it corresponds to a fixed dose of radiation delivered to the honeybees. We then excluded a study of a dose-effect relationship on mortality. The 2-h exposure was split into $\mathrm{ON}$ and $\mathrm{OFF}$ intervals so that the OFF exposure was greater than the ON exposure in an attempt to mimic the field situation.

In the groups subjected to the ON/OFF ratio $1: 3$, the statistical analyses indicates that exposure to radio waves had no effect on mortality, and no clear relationship between mortality and the $\mathrm{ON}$ duration was observed (see Figure 2). Increasing the $\mathrm{ON}$ duration from 1 to $60 \mathrm{~s}$ had no consequence on mortality whatever the HF or UHF exposure. Moreover, the same ON duration of $60 \mathrm{~s}$ had no effect in the condition $60 \mathrm{~s} / 180 \mathrm{~s}$ whereas it was associated with an effect of UHF in the condition $60 \mathrm{~s} / 300 \mathrm{~s}$ (1:5 ratio). As the total exposure and $\mathrm{ON}$ durations are identical in both conditions (see Table II), we may assume that the main factor explaining the increased mortality in the second case could be the splitting ratio and, specifically, the OFF duration, equal to $5 \mathrm{~min}$. Increased HF mortality was also associated with the condition $120 \mathrm{~s} / 360 \mathrm{~s}$, corresponding to OFF duration of $6 \mathrm{~min}$. This is an unexpected result, as logical reasoning would predict that OFF durations protect the animals. It might be that continuous radiations or radiations with short interruptions induce an adaptive process whereas spaced out radiations produce a contrasting effect with destructive consequences for the organism. In the framework of our experiments, this hypothesis could be tested by using a constant $\mathrm{ON}$ duration paired with OFF durations of increasing values in independent experiments, as was done in conditions $60 \mathrm{~s} / 180 \mathrm{~s}$ and $60 \mathrm{~s} / 300 \mathrm{~s}$.

Finally, in two conditions (HF, condition $120 \mathrm{~s} /$ $360 \mathrm{~s}$; UHF, condition $60 \mathrm{~s} / 300 \mathrm{~s}$ ) out of five, we observed an effect of exposure to radio waves on mortality. To precisely answer the question raised at the beginning of this work, which is the safety of the HF radiations emitted by the reader at the entrance of the hive, we report a single negative effect when honeybees are subjected to HF for $2 \mathrm{~h}$ split into $2 \mathrm{~min}$ ON and 6 min OFF.

Percentages of mortality observed in control groups remained under the value of $10 \%$ admitted in ecotoxicological studies (OECD 1998), except for the condition $1 \mathrm{~s} / 3 \mathrm{~s}$ for which the value was $12 \%$. We looked for a relationship between the number of honeybees gathered by cage and the mortality rate in the control groups, as honeybees' survival depends on the size of the group (Dechaume Moncharmont et al. 2003). For the condition $1 \mathrm{~s} / 3 \mathrm{~s}$, the size of the control group in each replicate was around 40 animals whereas the smallest size of the control groups was for the condition 1:5 (around 24 honeybees per replicate; see Table II) and was not associated with high mortality. Thus, the mortality of the controls in the condition $1 \mathrm{~s} / 3 \mathrm{~s}$ is not linked to the number of individuals per cage. This condition was performed early June, whereas the other conditions were performed successively during the summer. The fluctuations in mortality rates could be explained by uncontrolled parameters such as 
weather variations to which the hive was subject outside the laboratory or genetic factors determining the "quality" of the eggs. Actually, each replicate of each condition corresponds to a cohort of emergent honeybees. It could be that the cohorts were not strictly identical from a biological point of view due to different genetic backgrounds coming from the males that fertilized the queen. This could induce a biological variation in mortality visible as higher mortality early June.

The experimental conditions of laboratory radio wave exposure used in this study defined a worst condition: The 2-h exposure duration resulted from more than doubling the time spent by each honeybee beneath the readers in field experiments. Also, the exposure spreading was concentrated in time, going from 15 days in the field to 8 $12 \mathrm{~h}$ in the lab (see Table II). The effect of the exposure also depends on the distance between the radio wave source and the target. This distance is $2 \mathrm{~mm}$ in the field but varied from 0 to $8 \mathrm{~cm}$ in the lab (height of the plastic cage). We then estimate that the radiations received in the lab conditions are lower on the whole than in the field conditions, for comparable exposure durations. However, for the same distance, higher power density is associated with UHF than with HF (see Table I). In this sense, using UHF generators to diminish the constraint of the short distance between the honeybee and the reader raises the problem of the higher power received by the insects.

We measured the power densities of a cell phone $(900 \mathrm{MHz})$ in "search" mode $\left(450 \mathrm{nW} / \mathrm{m}^{2}\right)$ and in "connection" mode $\left(4 \mu \mathrm{W} / \mathrm{m}^{2}\right)$ and observed that the values are in between those measured for $13.56-$ and $868-\mathrm{MHz}$ radio waves (see Table I). Several studies have reported the effects of cell phone radiations on insect biology or strength of the hive, but the physical parameters of the radio waves (frequency, power density, field strength) or the exposure conditions are so variable that comparisons are difficult. For example, sub-lethal effects of radio waves were evaluated by Harst et al. (2006) and Kimmel et al. (2007) by exposing honeybees to $1900-\mathrm{MHz}$ radiation emitted by a Digital Enhanced Cordless Telephone-base station set at the base of the hive. A tendency to reduced honeycomb building and increased homing flight duration was noticed in exposed honeybees compared to non-exposed animals. These observations have to be taken cautiously as the authors did not indicate the exposure duration and did not control the age of honeybees, a critical factor. More recently, Favre (2011) introduced into the hive a mobile phone (900 $\mathrm{MHz}$, energy absorption rate $<2 \mathrm{~W} / \mathrm{kg}$ ) playing a radio station program for several hours and observed the induction of worker piping, a behaviour associated with disturbance of the hive. Although control experiments included hives without mobile phones or with mobile phones kept in standby mode, no control of the effect of the sounds emitted by the mobile phone was done. We cannot rule out that airborne sound signals perceived by honeybees (Hrncir et al. 2006) could induce them to produce piping in return. Negative effects on reproduction have been observed in Drosophila exposed to mobile phone radiations $\left(900 \mathrm{~Hz} ; 4360 \mathrm{~mW} / \mathrm{m}^{2} ; 37\right.$ $\pm 7 \mathrm{~V} / \mathrm{m}$ ) for 4 days, $6 \mathrm{~min} /$ day (Panagopoulos et al. 2004). The egg laying rate of the honeybees' queen and the strength of the hive were negatively affected by mobile phone radiations (900 MHz, $8.54 \mu \mathrm{W} / \mathrm{cm}^{2}$ ) delivered $1 \mathrm{~h} /$ week, for 12 weeks. In these two last examples, the level of radiations is higher $(37 \mathrm{~V} / \mathrm{m})$ and the duration of exposure $(12 \mathrm{~h})$ is longer than those we used. These experimental differences can explain the negative effects reported by the authors.

In conclusion, our results show that exposure to HF radio waves increases mortality in one condition (out of five) that strongly maximizes the exposure conditions in the field experiments. We can reasonably admit that the hazard would be even more reduced if the exposure duration remained inferior to $2 \mathrm{~h}$ per day. We are aware that more experiments controlling several exposure factors (nature of the radiation source, distance from the source of radiation, duration and distribution in times of the radiations) are needed before firmly drawing conclusions on the effects of radio waves on honeybee health. 


\section{ACKNOWLEDGMENTS}

This work was supported by a grant from the European Community Program for French beekeeping coordinated by the French Ministry of Agriculture, in association with the CNRS and the French Ministry of Research and Higher Education. We are very grateful to Gaëtan Nozet and Jean-François Bompa who helped us in measuring power densities of the HF and UHF radiations. Thanks to Jean-Louis Druilhe and Patrick Arrufat for sharing with us their knowledge on electromagnetic radiations. The authors also thank Ilan Gauthier for his help in the design of Figure 1 and Pauline Haas-Hammel for proofreading.

Effets des radiations à haute fréquence sur la survie des abeilles (Apis mellifera)

radiation électromagnétique / environnement / santé / insecte / Apidae

Auswirkungen von Hochfrequenzradiowellen auf das Überleben von Honigbienen (Apis mellifera $\mathbf{L}$.)

Elektromagnetische Strahlung / Umwelt / Gesundheit / Insekt

\section{REFERENCES}

Capaldi, E.A., Dyer, F.C. (1999) The role of orientation flights on homing performance in honeybees. J. Exp. Biol. 202, 1655-66

Cucurachi, S., Tamis, W.L.M., Vijver, M.G., Peijnenburg, W.J.G.M., Bolte, J.F.B., de Snoo, G.R. (2013) A review of the ecological effects of radiofrequency electromagnetic fields (RF-EMF). Environ. Int. 51, 116140

D'Andrea, J.A., Adair, E.R., de Lorge, J.O. (2003) Behavioral and cognitive effects of microwave exposure. Bioelectromagnetics 24 (Suppl 6), S39-S62

Dechaume Moncharmont, F.X., Decourtye, A., HennequetHantier, C., Pons, O., Pham-Délègue, M.H. (2003) Statistical analysis of honeybee survival after chronic exposure to insecticides. Environ. Toxicol. Chem. 22, 3088-94

Decourtye, A., Devillers, J., Aupinel, P., Brun, F., Bagnis, C., Fourier, J., Gauthier, M. (2011) Honeybee tracking with microchips: a new methodology to measure the effects of pesticides. Ecotoxicology 20, 429-437

Favre, D. (2011) Mobile phone-induced honeybee worker piping. Apidologie 42, 270-279
Feltham, H., Park, K., Goulson, D. (2014) Field realistic doses of pesticide imidacloprid reduce bumblebee pollen foraging efficiency. Ecotoxicology 23, 317-323

Gill, R.J., Raine, N.E. (2014) Chronic impairment of bumblebee natural foraging behaviour induced by sublethal pesticide exposure. Funct. ecol. 28, 1459-1471

Gill, R.J., Ramos-Rodriguez, O., Raine, N.E. (2012) Combined pesticide exposure severely affects individualand colony-level traits in bees. Nature 491, 105-108

Harst W., Kuhn J., Stever H. (2006) Can electromagnetic exposure causes a change in behaviour? Studying possible non-thermal influences on honeybees - An approach within the framework of educational informatics. Acta Systemica - IIAS Int J 1:1-6. Available:http:// www.hese-project.org/hese-uk/en/papers/ icrw_kuhn_landau_study.pdf Accessed 17 march 2015

Henry, M., Beguin, M., Requier, F., Rollin, O., Odoux, J.F., Aupinel, P., Aptel, J., Tchamitchian, S., Decourtye, A. (2012) A common pesticide decreases foraging success and survival in honeybees. Science 336, 348-350

Henry, M., Bertrand, C., Le Féon, V., Requier, F., Odoux, J.F., Aupinel, P., Bretagnolle, V., Decourtye, A. (2014) Pesticide risk assessment in free-ranging bees is weather and landscape dependent. Nature Commun. 5, 4359-4366

Hrncir, M., Barth, F. G., Tautz, J. (2006) Vibratory and Airborne Sound-Signals in Bee Communication. In: S. Drosopoulos and M. Claridge (eds.), Insect Sound and Communication: Physiology Behaviour, Ecology, and Evolution, pp. 421-436. CRC Press, Taylor \& Francis Group, Boca Raton, London, New York

Kimmel S., Kuhn J., Harst W., Stever H. (2007) Electromagnetic radiation: influences on honeybees (Apis mellifera ). Preprint IIAS - InterSymp Conference, Baden-Baden. Available: http://www.hese-project.org/ hese-uk/en/heseuk/profile.php?id=hst. Accessed 17 march 2015

Krewski, D., Glickman, B.W., Habash, R.W., Habbick, B., Lotz, W.G., Mandeville, R., Prato, F.S., Salem, T., Weaver, D.F. (2007) Recent advances in research on radiofrequency fields and health: 2001-2003. J. Toxicol. Environ. Health B Crit. Rev. 10, 287-318

Molet, M., Chittka, L., Stelzer, R.J., Streit, S., Raine, N.E. (2008) Colony nutritional status modulates worker responses to foraging recruitment pheromone in the bumblebee Bombus terrestris. Behav. Ecol. Sociobiol. 62, 1919-1926

OECD (1998) Test No. 213: Honeybees, Acute Oral Toxicity Test. OECD Guidelines for the Testing of Chemicals, Section 2, OECD Publishing. doi: 10.1787/9789264070165-en

Panagopoulos, D.J., Karabarbounis, A., Margaratis, L.H. (2004) Effect of GSM 900-MHz mobile phone radiation on the reproductive capacity of Drosophila melanogaster. Electromagnetic Biol. Med. 23, 29-43

Schneider, C.W., Tautz, J., Grünewald, B., Fuchs, S (2012) RFID tracking of sublethal effects of two neonicotinoid insecticides on the foraging behavior of Apis mellifera. PLoS ONE. 7, e30023 
Sharma, V.P., Kumar, N.R. (2010) Changes in honey bee behaviour and biology under the influence of cell phone radiations. Curr. Sci. 98, 1376-1378

Stelzer, R.J., Chittka, L. (2010) Bumblebee foraging rhythms under the midnight sun measured with radiofrequency identification. BMC Biol. 8, 93-99

Streit, S., Bock, F., Pirk, C.W.W., Tautz, J. (2003) Automatic life-long monitoring of individual insect behavior now possible. Zoology 106, 169-171

Sumner, S., Lucas, E., Barker, J., Isaac, N. (2007) Radio-tagging technology reveals extreme nestdrifting behavior in a eusocial insect. Curr. Biol. 17, 140-145
Vanderstraetan, J., Verschaeve, L. (2008) Gene and protein expression following exposure to radiofrequency fields from mobile phones. Environ. Health Perspect. 116, 1131-1135

Warnke U. (2007) Bees, Birds and Mankind - Destroying nature by 'electrosmog'. In: Hecht K, Kern M, Richter $\mathrm{K}$, Scheiner HC (Eds), Effects of Wireless Communication Technologies. Competence Initiative for the Protection of Humanity, Environment and Democracy. Brochure 1, Kempten, Germany, pp 4-45

Wiholm, C., Lowden, A., Kuster, N., Hillert, L., Arnetz, B.B., Akerstedt, T., Moffat, S.D. (2009) Mobile phone exposure and spatial memory. Bioelectromagnetics 30, 59-65 